\title{
Abnormality of visual neuromagnetic activation in female migraineurs without aura between attacks
}

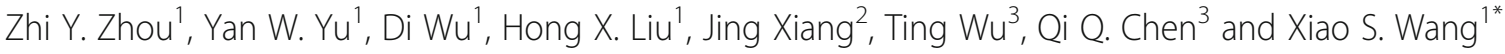

\begin{abstract}
Objective: The present study aimed to preliminary explore the abnormal neuromagnetic activation in female migraine patients between attacks using magnetoencephalography (MEG) and pattern reversed visual evoked magnetic fields (PR-VEFs).
\end{abstract}

Methods: A total of 17 female migraine subjects during the headache-free phase and 17 healthy controls (HC) were studied using a 275-channel magnetoencephalography (MEG) system. In this study, visual evoked magnetic fields (VEFs) were generated by a pattern-reversal check as the visual stimulus. The average of $100 \mathrm{VEFs}$ was evolved by different half patterns were averaged and used to analyze waveform, spectrum, and source location within two frequency ranges $(5-100$ and $100-1000 \mathrm{~Hz})$, respectively.

Results: In migraine subjects, the latency of second peak of VEFs (VIIs) showed significant prolongations when compared with HC. On the sensor level, the cortical spectral power in migraine subjects was similar to that of $\mathrm{HC}$ in the $5-100 \mathrm{~Hz}$ range and was lower in the $1000-1000 \mathrm{~Hz}$ range. There was a decrement of source strength in the visual cortex in migraine patients when compared to HC in both the 5-100 and 100-1000 Hz frequency range. Moreover, there was a similar odds of activation in 5-100 and 100-1000 Hz frequency ranges in the area beyond the primary visual cortex between the two groups. In addition, no correlation was observed between clinical data (intensity of headache, headache-history duration, the frequency of headaches) and MEG results.

Conclusions: The findings presented in the current study, suggested that interictal cortical activation following a visual stimulus was low in female migraine patients. The low pre-activation was detected in the visual cortex using VEF and MEG in both low and high-frequency band. Our results add to the existing evidence that cortical interictal excitability change may be relative to the pain-module mechanism in migraine brains. Thus, our data improved the apprehension of the cortical disorder of migraine in the high-frequency domain.

Keywords: Migraine, Visual evoked magnetic fields, Magnetoencephalography

\section{Background}

Migraine is a common, disabling neurological disorder, clinically manifested by episodes of moderate to severe episodic pain that are accompanied by autonomic nervous system dysfunction [1]. As one of the 20 most disabling diseases [2], migraine exerts a huge impact on individuals and society, and is considered an important public health problem. It has been estimated that

* Correspondence: lidou2005@126.com; zzyxx@vip.qq.com

${ }^{1}$ The Department of Neurology, Nanjing Brain Hospital, Nanjing Medical University, Guang Zhou Road 264, Nanjing, Jiangsu 210029, People's Republic of China

Full list of author information is available at the end of the article approximately $31 \%$ of migraine patients have missed at least $24 \mathrm{~h}$ of work or school in the latest 3 months because of migraine, and $51 \%$ of patients has reported at least a $50 \%$ reduction in productivity at work or school [3]. The prevalence of migraine is different between the male and female population, and according to multiple surveys, the prevalence of migraine in females is 3-fold higher compared to that in males in the general population $[4,5]$.

Migraine has been considered a primary disorder of the brain, not a primary vascular event $[1,6]$. Recent reports have shown that dysfunctions of the cerebral cortex and 
brain-stem or diencephalic are fundamental in the underlying mechanism of migraine [6, 7]. Among many cortical regions, the occipital cortex has been an obvious region of interest regarding the functional change in the brain of a migraine patient for many reasons. Firstly, approximately $30 \%$ of migraineurs have transient neurological symptoms that are most frequently visual (MA) preceding headache attacks [8], second, most (60\%) migraineurs suffer from distinctive visual disturbance before or during a headache attack [9]. In addition, exposing interictal migraineurs to light may trigger the initialization of a headache [10]. Albeit the essential role the visual cortex may plat in the pathophysiology of migraine, electrophysiological experiments have failed to consistently conclude on the changes in excitability of migraine brains throughout the periodic stage of migraine $[11,12]$, the cerebral mechanisms underlying such differences remain unclear.

The visual evoked potential (VEP) is an acknowledged neurologic diagnostic tool for detecting disease-induced changes in visual pathways or visual cortical function. VEP studies have a domesticated deficiency in cortical habituation ability in individuals with migraines, which is evidenced by failing to decrement in amplitude over time [13, 14], and shows cortical hyperresponsivity of the visual cortex. Magnetoencephalography (MEG, an atraumatic neuroimaging modality for investigating functional activation in the brain with outstanding temporal resolution and satisfactory special resolution [15]. MEG receives neuromagnetic signals that penetrate the skull and scalp without distortion caused by the skull, skin, or other tissues [16]. Compatible with the triphasic deflection in VEPs, neuromagnetic activation elicited by the visual stimulus can be detected by MEG $[11,17]$. Among the components of PR-Visual Evoked Fields (VEF), P100 was considered the most reliable, robust activation, and therefore is widely investigated in VEF/VEP studies $[18,19]$. According to the anatomical organiza tion of the visual cortex, the left striate cortex or ex-striate cortex is activated by the stimulus on the right side of the visual field, and vice-versa (known as the Cruciform Model) [20, 21].

Previous reports in both VEP and VEF studies involving migraine subjects have shown conflicting results mainly due to methodological differences (i.e., check size, stimulus fields, reversal rate, etc.), and the patient's state during the experiment (e.g., the time interval to last attack, menstrual phase) [12, 22]. Unfortunately, these studies only focused on the waveform level in the comparatively low-frequency band $(<100 \mathrm{~Hz})$ [23]. Although high-frequency oscillations may reveal more information about the functional changes in cortical function in the human brain [24-26], the spatiotemporal characteristics of visual evoked neuromagnetic activation in migraine patients on a high-frequency level is mostly unclear.
In this study, we aimed to investigate the signature of aberrant visual evoked neuromagnetic activation on the level of different frequency ranges in female subjects with migraine without aura (MwoA) using MEG and a reversal checkboard pattern (PR-VEFs). To analyze the data, Morlet Wavelet analysis was used, instead of conventional band-pass filters and a waveform-based beamformer to demonstrate the frequency distribution of VEF. Unlike other VEP/VEFs studies, which focused on the central cortical excitability habituation of the migraine brain, we analyzed the visual neuromagnetic spectrograms on the waveform, sensor and source level at two main frequencies: $5-100 \mathrm{~Hz}$, and $100-1000 \mathrm{~Hz}$. We hypothesize that neuromagnetic activation evoked by visual stimuli in the female migraineur was aberrant from that of $\mathrm{HC}$, and this change could be identified at both low $(5-100 \mathrm{~Hz})$ and high-frequencies (100-1000 $\mathrm{Hz}$ ). To our knowledge, this is the first study that focuses on the visual evoked neuromagnetic field in female migraineurs in which MEG and a wide frequency range were used.

\section{Methodology \\ Subjects}

Twenty female patients (age: $35.2 \pm 6.8$ years) from the neurology outpatient department of Nanjing brain hospital (NBH) were enrolled in the present study. Inclusion criteria were as follows (i): Patients explicitly diagnosed with migraine without aura (MwoA) in accordance with the International Classification of Headache Disorders, 3nd Edition bete (ICHD-III beta) [27]; (ii): No medical history of other neurological or ophthalmological disorders (patients as well as $\mathrm{HC}$ ). (iii) No receipt of any pharmacological prophylactic treatment in 3 months prior to the study, such as valproate, triptans or ergot derivatives). $\mathrm{HC}$ matched migraine patients regarding age, gender, and education degree. $\mathrm{HC}$ were recruited from employees of the Nanjing brain hospital and their relatives or friends. Inclusion criteria for control group included: (i): Healthy individuals without a history of neuropsychiatric disorders, migraine or any other form of headache, brain injury, and any vision deficiency. Exclusion criteria for both patients and HC included: (i) The presence of any metal implant that might cause magnetic noise in the MEG data; (ii) Inability to remain still during the MEG recording. Migraine subjects were asked to be recorded during the interictal phase (at least 2 days before or after a migraine attack). Clinical characteristics of migraine subjects were accessed by a questionnaire prior to MEG recording, the contents of clinical assessment included: age, disease history, headache frequency (times/month), duration of headache attacks in the latest month, headache locus, and headache accompany symptoms, such as phonophobia, photophobia, nausea and 
vomiting, intensity of headache assessed by the Visual Analogue Scale (VAS).

\section{Visual stimulus paradigm}

A MEG scan was performed on all respondents while they were asked to stare at a fixed yellow dot in the middle of checkboard pattern on a screen that was placed approximately $32 \mathrm{~cm}$ in front of the subjects. Patients were asked to try limit blinking because blinking could result in noise in MEG data. Pattern-reversal checkerboard stimuli were generated using customized software, Brain X (Jing Xiang, Ohio, USA, Cincinnati children hospital) [28]. The visual stimulus consisted of three consecutive reversal patterns with a reversal rate of 1 $\mathrm{Hz}$. These patterns are sequentially presented as: full-field, left-field, and right-field, and the presence of each pattern was $600 \mathrm{~ms}$, with a $400 \mathrm{~ms}$ gap between patterns. The size of the checkboard was $60 \mathrm{~min}$ of arc, while extended $15(\mathrm{~W})^{*} 22(\mathrm{H})$ in the left hemifield of the subject with the mean luminance set at $12 \mathrm{~cd} / \mathrm{m}^{2}$, and contrast 0.94 . On the screen, there was a delay of 400 ms between trigger onset and stimulus presentation and this time lag was subtracted from the target time window. Visual task stimulus consisted of 100 triggers for each field type (left, right and full field) for one set of recording. The stimulus presentation and signal recording were accomplished with Brain X software, the 100 times of responses from different fields were automatically collected and averaged by the software. Each set lasted for about $5 \mathrm{~min}$, each participant was asked to complete 2 sets of tasks.

\section{MEG recording}

For each participant, the MEG signal was recorded in a controlled magnetic-shielded room by integration of a whole-head 275 channel (CTF, Canada). All patients were requested to remove any magnetic materials from their bodies. There were attachments of electromagnetic coils (Fiducial markers) at the left and right preauricular points and at the nasion point to measure the patients head position in comparison to the sensors of MEG. During the entire scanning procedure, participants were requested to lay in a comfortable position with both arms resting on each side For each channel, MEG record was digitized at $6000 \mathrm{~Hz}$. The acquisition window was set at $1000 \mathrm{~ms}$ for each trial, and the $400 \mathrm{~ms}$ after the trigger (presence of the reversal pattern) was recorded in the MEG system. MEG data were then recorded by the system after a third-order gradient noise cancellation process. The threshold of permitted head movement during MEG scanning was $5 \mathrm{~mm}$. If head movement during one scan was beyond $5 \mathrm{~mm}$, the dataset was disregarded, and a new scan was recorded.

\section{Magnetic resonance imaging scan}

All participants in this study underwent three-dimensional magnetic resonance imaging (MRI) on a 1.5 MRI (Singa, GE, USA). In brief, three fiduciary marks were placed at locations that were identical to the position of the three coils used in the MEG recordings for the co-registration of the MRI data and MEG data sets. Subsequently, all anatomic landmarks were made identifiable in the MRIs.

\section{Data analysis Morphology}

MEG data from all participants were manually analyzed using a custom-designed program, MEG Processor. In brief, data were firstly processed by removal of the Direct Current (DC) base on the results on a pre-trigger baseline. Subsequently, a low pass $(5 \mathrm{~Hz})$ and a high pass filter $(100$ $\mathrm{Hz}$ ) were applied to obtain the morphology VEFs. The latency of the magnetic fields was measured by putting the cursor on each of the peaks of neuromagnetic components. At least three neuromagnetic components were identified, namely VI, VII, and VIII. In the present study, the quantification of waveforms following the visual stimulus focused on VII.

\section{Time-frequency analysis}

MEG waveforms information was transformed to spectrograms using the Morlet continuous wavelet transform. The average of all trials prior to the transformation was taken, therefore spectrograms are presented as averaged time-frequency domains [29-31]. The spectral characteristics of MEG data were analyzed with spectrograms computed with Morlet continuous-wavelet algorithm using the following equation:

$$
w(t, s)=C_{\sigma} \pi^{-\frac{1}{4}} e^{-\frac{1}{2} t^{2}}\left(e^{i \sigma t}-k_{\sigma}\right)
$$

Because frequency-temporal resolution changes with the sigma value, this study dynamically changes sigma value (the number of wave circles) according to frequency ranges [30,31]. In our previous study, we showed that to achieve a higher time sensitivity in $5-100 \mathrm{~Hz}$ and higher frequency sensitivity in $100-1000 \mathrm{~Hz}$ range, the sigma values were considered 1,3 for the 5 to $100 \mathrm{~Hz}$ and 100 to $1000 \mathrm{~Hz}$ range, respectively, with frequency bins set at $600 \mathrm{~Hz}$ [30, 31]. Specifically, neuromagnetic signals below $5 \mathrm{~Hz}$ were not included in the analysis because a lengthier time window was required for the computation of low-frequency components of the data, however in the present study, we only focused on time domain in 0-200 $\mathrm{ms}$ following the visual trigger, which included all VEF-related components. Neuromagnetic signals above $1000 \mathrm{~Hz}$ were also excluded from this study because in our pre-analysis these were not captured at the very-high-frequency range $(>1000 \mathrm{~Hz})$. The selection of 
the frequency ranges was based on our previous findings $[29,31,32]$.

\section{Sensor-level analysis}

To better illustrate the source patterns of visual-evoked neuromagnetic activation, the spatial characteristics of visual-evoked neuromagnetic activation at the sensor level were estimated through polarity contour maps [29, 33]. To quantify the strength of activation, the Root-MeanSquare (RMS) value from all sensors was measured to represent the absolute spectral power. All measurements were obtained by a toolkit that automatically obtained the mean and peak value for each frequency bin of all MEG sensors. This approach was employed because the entire calculation could be objectively completed by a computer. Moreover, the quantization spectral power at sensor levels was time-locked at the VIIs activation for all subjects and frequency ranges. For all frequency ranges, the temporal resolution was 6 data points per millisecond. The frequency bands for quantifying spectral power at sensor levels is for 5 to $100 \mathrm{~Hz}$. All spectral power in different frequency bands was normalized mathematically, and the spectral power of a selected region was divided by the number of frequency bins, therefore, the data were comparable with the findings presented in other studies using a different frequency resolution.

\section{Source analysis}

Neuromagnetic sources were localized with volumetric source imaging [34]. In the relatively new method, each coordinate was scanned using a voxel with a 4-mm spatial resolution. In this study, we focused on the VIIs waveform from all subjects because the different components of VEFs have different generators in the visual cortex [35]. Thus, a fixed window was applied for activating VEF. A MEG processor was used for analyzing magnetic sources. According to the cruciform model mentioned above, the dominant activation of VIIs was around the primary visual area (V1), several other visual-related regions may also be activated. The computation was separately performed in two frequency ranges from 5 to $100 \mathrm{~Hz}$ and 100 to $1000 \mathrm{~Hz}$.

\section{Statistical analysis}

Latency, spectral power, and source strength of VEFs between migraine subjects and $\mathrm{HC}$ were analyzed by Student $\mathrm{t}$-tests. All data were tested by normlity tests (K-S test) before Student $\mathrm{t}$-tests. The relevance between parameters of VEFs and clinical characteristics (intensity of headache, headache-history duration, and the frequency of headache) was analyzed with Pearson correlation test. The difference of odds ratios of activation following visual stimulus in brain areas other than the contralateral primary visual cortex (cPVC) between migraineurs and controls were analyzed by the Fisher exact test. For all participants, statistical analyses were performed twice for the right half and left hemisphere separately. The threshold of statistical significance for differences was set at $p<0.05$. Bonferroni correction was not applied because there was no multiply comparison for the data.

\section{Results}

\section{Clinical characteristics}

The demography characteristics of migraine patients and $\mathrm{HC}$ are shown in Table 1. Out of the 17 patients with migraine, all migraine (100\%) subjects suffered from migraine without aura (MwoA), and 9 subjects showed photophobia during the ictal period (the other 8 subjects did not show photophobia during the ictal period). All subjects (100\%) were right-handed, 8 subjects (47\%) had headache bilaterally; 6 migraine subjects (35\%) had unilateral headache attacks on the left supraorbital areas and 3 migraineurs (17\%) on the right supraorbital areas. No subjects reported any headache attack triggered by visual stimulus during the recordings.

\section{Waveform analysis}

Data were bandpass filtered at $5-100 \mathrm{~Hz}$ for demonstration of the waveform. The morphology of waveforms is shown in Fig. 1. In the $5-100 \mathrm{~Hz}$ range, the latency of VIIs from migraine subjects was significantly prolonged when compared with $\mathrm{HC}$ following both left and right visual stimulus. Significant differences were found between the latency of VIIs evoked by different half stimulus within the migraine group and $\mathrm{HC}$ group. Notably, the conventional measurement of amplitudes was not included in this observation because in this study source strength was used to better estimate the activation power.

Table 1 Clinical Characteristics of Subjects

\begin{tabular}{lll}
\hline Parameters & Migraine & Control \\
\hline Gender (female/male) & $0 / 17$ & $0 / 17$ \\
Age (years) (mean \pm SD) & $33.6 .2 \pm 4.5$ & $32.1+3.1$ \\
Handedness, right/left & $0 / 17$ & $0 / 17$ \\
Years of migraine (mean \pm SD) & $9.8 \pm 6.4$ & NA \\
Frequency of headache per month (mean \pm SD) & $1.6 \pm 1.4$ & NA \\
Severity of headache (VAS scale) & $6.6 \pm 1.4$ & NA \\
Prophylactic treatments in recent three months & $0 / 17$ & NA \\
Pain type (number of subjects; multiple descriptions were allowed) \\
Throbbing & 12 & NA \\
Pressure & 2 & NA \\
Constant & 3 & NA \\
Sharp & 1 & NA \\
Squeezing & 2 & NA \\
Stabbing & 2 & NA \\
Others & 0 & NA \\
\hline
\end{tabular}

Participants may have more than 1 type of pain Abbreviations: N/A Not available 


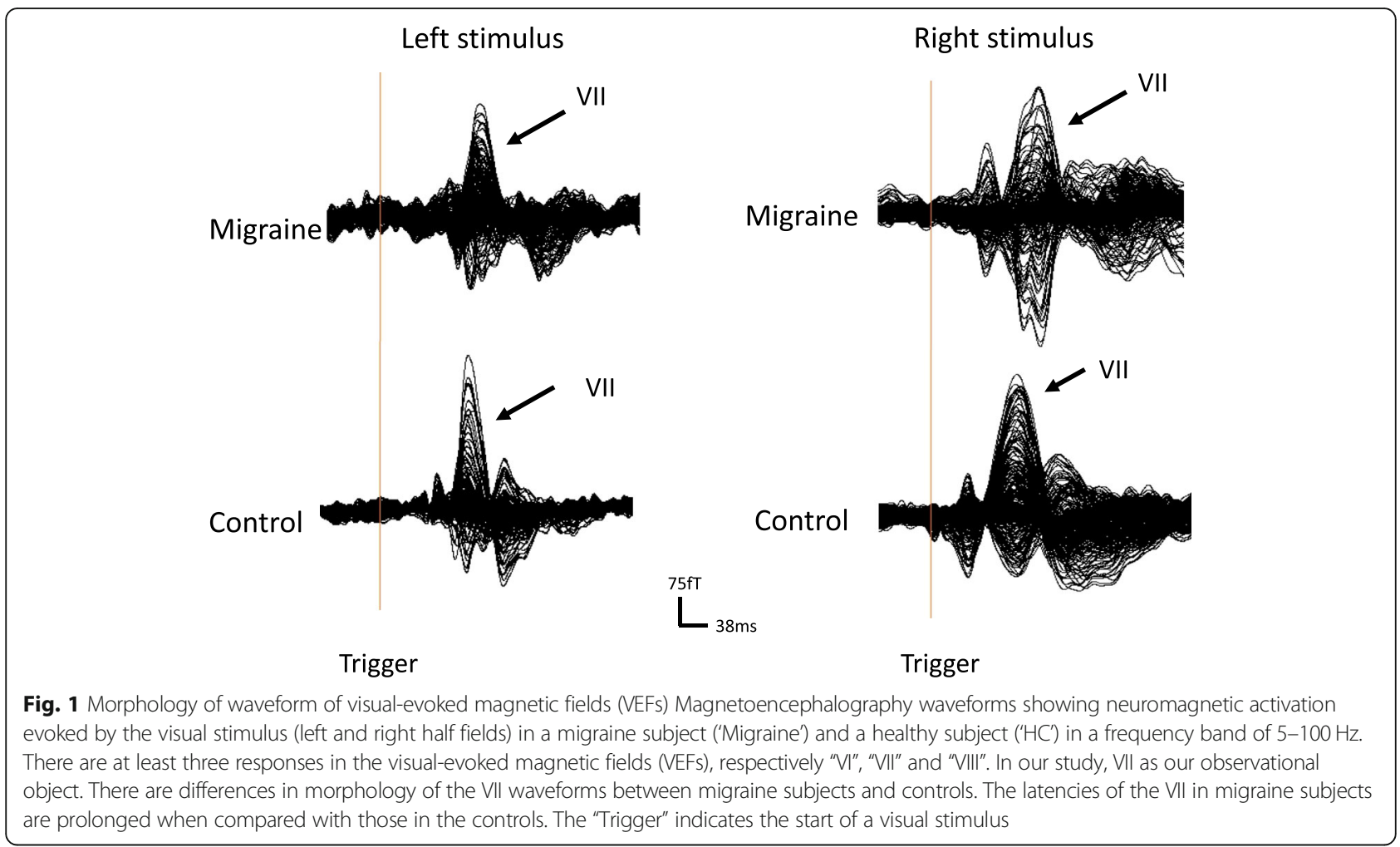

\section{Time-frequency analysis}

In all migraine subjects (17/17) and all $\mathrm{HC}(17 / 17)$ there were at least three oscillatory components revealed by the polarity spectrograms in the frequency range of $5-100 \mathrm{~Hz}$, especially in $5-30 \mathrm{~Hz}$ (see Fig. 2). To quantify the neuromagnetic spectral power, RMS was obtained to access "global spectral power" by calculating the sum of the spectral power from all sensors over the target frequency ranges of 5 to $100 \mathrm{~Hz}$. Interestingly, no significant differences were observed in averaged spectral power in the frequency range of $5-100 \mathrm{~Hz}$ between migraine subjects and $\mathrm{HC}$. The source patterns were analyzed with spectral contour maps, which showed that the activation of neuromagnetic responses was localized in the contralateral primary visual cortex in both migraine subjects and $\mathrm{HC}$, and the odds of activation in regions beyond primary visual cortex (i.e. extensive visual cortex; parietal/temporal regions) was similar between migraine subjects and $\mathrm{HC}$ in $5-100 \mathrm{~Hz}$. The values of spectrograms from all MEG sensors showed more interindividual variation among migraine subjects when compared to $\mathrm{HC}$.

Polarity spectrogram showed focal increased activation in the contralateral visual area represented by the lightning color bar in $100-1000 \mathrm{~Hz}$ (see Fig. 3), especially in $100-300 \mathrm{~Hz}$ in 9 of the migraine subjects $(9 / 17)$ and $8 \mathrm{HC}(8 / 17)$ especially in the $100-300 \mathrm{~Hz}$ range. In addition, activation was observed in regions outside of the visual cortex, including the contralateral/ipsilateral temporal lobe. Our data showed that spectral power estimated by RMS in migraine subjects showed a decrement compared to $\mathrm{HC}$ in this frequency range, while the odds of activation in these regions was similar between migraine subjects and $\mathrm{HC}$.

\section{Source analysis}

In the $5-100 \mathrm{~Hz}$ bandpass, all subjects showed activation in the contralateral visual cortex during stimulated by the right-field and left-field stimulus (17/17 vs $17 / 17)$. Activation was observed in other brain regions beyond the contralateral primary visual cortex (RBcPVC), these regions distributed from the associated visual cortex to parietal/temporal areas. Among all subjects analyzed using MSI, 7 migraine subjects and $3 \mathrm{HC}$ showed activation in the RBcPVC during the left-field stimulus (8/17 vs $5 / 17)$ and 7 migraineurs and $5 \mathrm{C}(7 / 17$ vs 5/17) during right-field stimulus (see Fig. 4 and Table 2). No significant differences were observed between the odds of activation in $\mathrm{RBCPVC}$ following either right or left visual stimulus $(p>0.05)$. The source strength of activation in migraine subjects was lower when compared to that of $\mathrm{HC}$.

In the $100-300 \mathrm{~Hz}$ frequency band, MSI showed that for 11 migraineurs and $9 \mathrm{HC}$ activation was mainly localized in the contralateral primary visual cortex (cPVC) $(11 / 17$ vs 9/17) during left-field stimulus, and in 11 migraineurs, and $10 \mathrm{HC}$ during right-field stimulus, while the neuromagnetic activation of $\mathrm{cPVC}$ was silent in the 


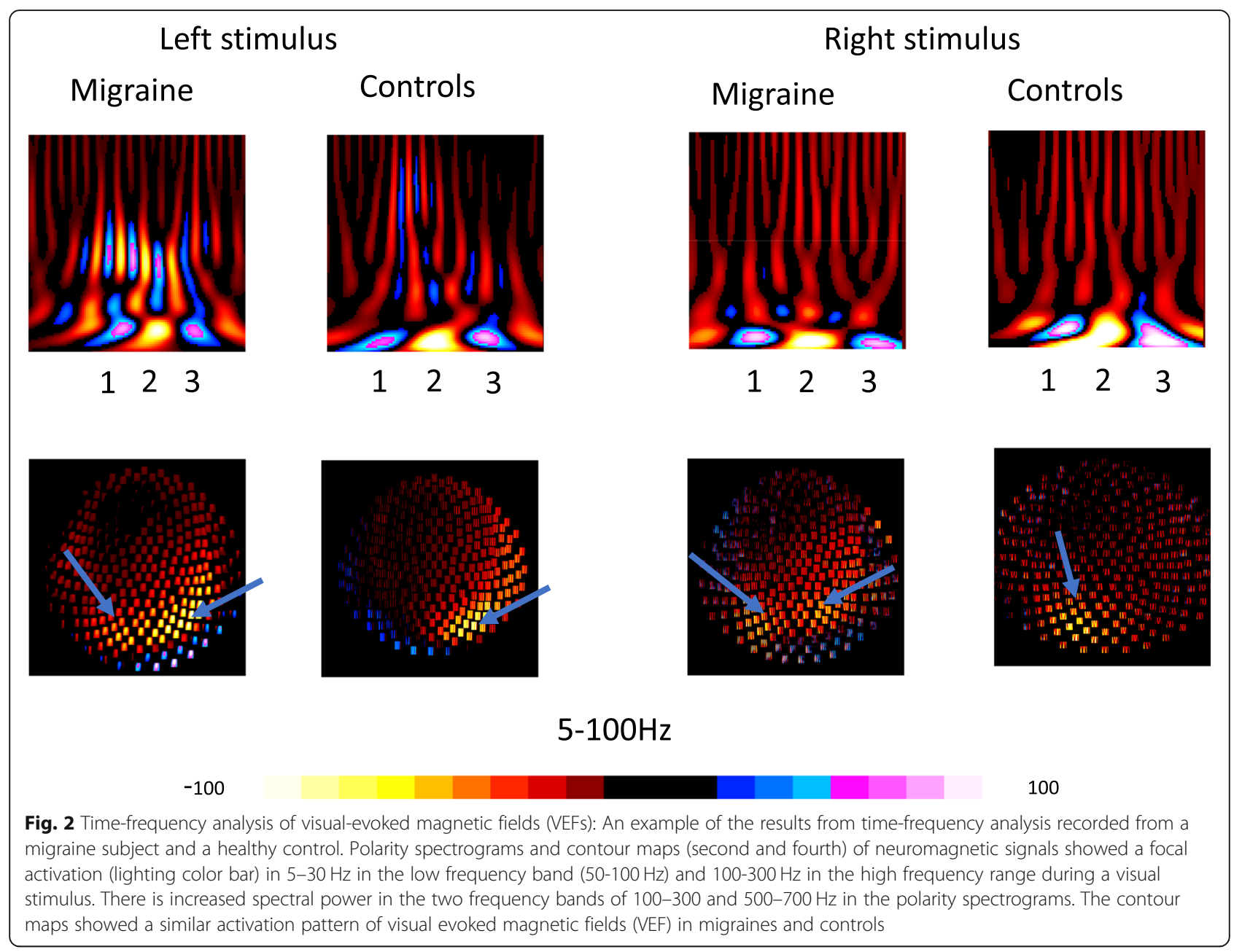

remainder of the participants. Moreover, 6 migraineurs and $7 \mathrm{HC}$ (6/17 vs 7/17) showed activation in RBcPVC following left-field stimulus, and 3 migraineurs and $5 \mathrm{HC}$ during the right $(3 / 17$ vs $5 / 17)$. Similar to that in low-frequency band, the odds of activation in RBCPVC was similar between migraine subjects and $\mathrm{HC}$ for both the left and right field. In this frequency band, the strength of activation on the source level in migraine subjects was significantly lower when compared to that of $\mathrm{HC}$ following both left and right-field stimulus $(p<0.05)$ (see Fig. 4 and Table 2).

\section{Neuromagnetic correlations of clinical characteristic}

Analysis of the correlation between latency and clinical data revealed that the latency of VIIs activated by left or right-field pattern did not correlated with the frequency of headaches per month, or the severity of headache or the years of migraine. No significant differences were observed between Latency, spectral power (RMS), source strength and these clinical data in either left or right hemisphere.

\section{Discussion}

Our study preliminarily explored the abnormal pattern reversal-visual evoked magnetic fields (PR-VEFs) in female migraine patients during the interictal period in the $5-1000 \mathrm{~Hz}$ frequency range. Our data demonstrated that interictal female migraineurs showed aberrant neuromagnetic visual responses that can be detected by MEG and PR-VEFs in both the low and high-frequency range. By adopting measurements of the waveform, spectrogram, and source localization methods in different frequency bands, it was found that the latency of neuromagnetic responses was significantly prolonged when compared to those in $\mathrm{HC}$, while the spectral power regarding cortical activation and odds of activation in other regions besides the primary visual cortex was similar in the low-frequency range $(5-100 \mathrm{~Hz})$. In the high-frequency range $(100-1000 \mathrm{~Hz})$, however, the activation strength of the neuromagnetic response was lower when compared to that of $\mathrm{HC}$, and the odds of neuromagnetic activation in regions other than contralateral primary visual cortex was similar as in low- 

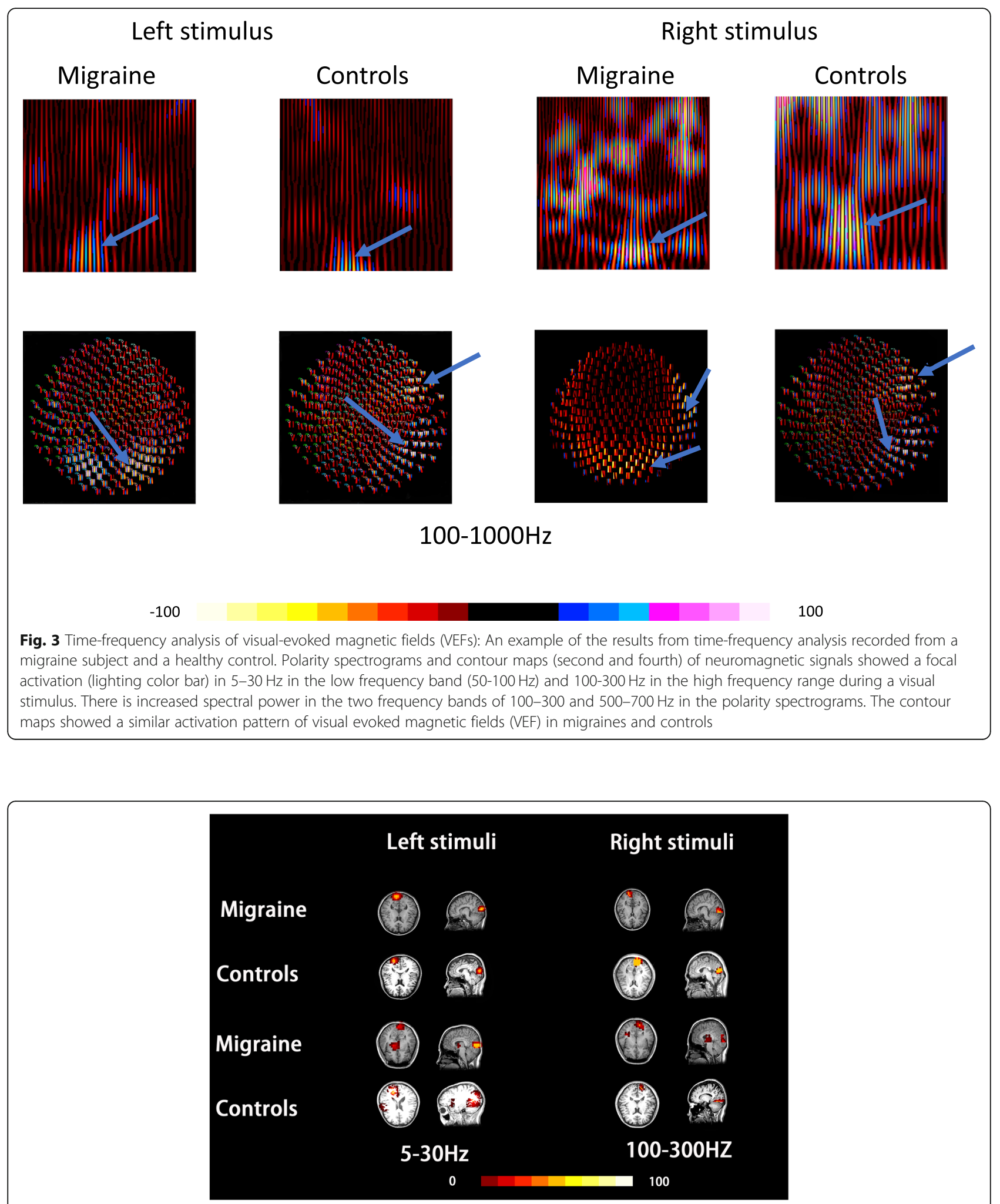

Fig. 4 Source location of V2: Magnetic source imaging (MSI) shows the source of activation of neuromagnetic response evoked by a visual stimulus in 5-30 and 100-300 Hz frequency bands in a migraine subject (migraine) and a control (control). The neuromagnetic activation evoked by a visual stimulus is mainly localized in the contralateral primary visual cortex (CPVC) in all migraineurs and controls, activation in regions beyond contralateral primary visual cortex (RBCPVC) was also noticed in some of the migraineurs and controls. Those areas included extensive visual cortex and parietal/temporal areas 
Table 2 The main dependent variables analyzed in the MEG spectrograms for migraine subjects and controls

\begin{tabular}{|c|c|c|c|c|c|}
\hline Frequency range & dependent variable & Position of the pattern & Migraine subjects & Healthy controls & $P$ value \\
\hline \multirow[t]{10}{*}{$5-100 \mathrm{~Hz}$} & \multirow[t]{2}{*}{ Latency } & Left & $122.2 \pm 4.9$ & $116.2 \pm 5.1$ & 0.002 \\
\hline & & Right & $113.7 \pm 5.3$ & $108.8 \pm 4.1$ & 0.006 \\
\hline & \multirow[t]{2}{*}{ Spectral Power (RMS) } & Left & $115.4 \pm 11.0$ & $139.5 \pm 6.2$ & $<0.00$ \\
\hline & & Right & $92.5 . \pm 8.9$ & $71.1 \pm 8.6$ & $<0.00$ \\
\hline & \multirow[t]{2}{*}{ Source Strength (fT/Hz) } & Left & $355.3 \pm 21.8$ & $370.0 \pm 16.3$ & 0.035 \\
\hline & & Right & $258.2 \pm 13.5$ & $262.7 \pm 10.0$ & NS \\
\hline & \multirow[t]{4}{*}{ Location } & \multirow[t]{2}{*}{ Left } & CPVC: $(17 / 17)$ & CPVC: (17/17) & NS \\
\hline & & & RBCPVC: (8/17) & RBCPVC: (5/17) & NS \\
\hline & & \multirow[t]{2}{*}{ Right } & CPVC: (17/17) & CPVC: (17/17) & NS \\
\hline & & & RBCPVC: (7/17) & RBCPVC: (5/17) & NS \\
\hline \multirow[t]{6}{*}{$100-1000 \mathrm{~Hz}$} & \multirow[t]{2}{*}{ Source Strength (fT/Hz) } & Left & $17.4 \pm 3.2$ & $21.1 \pm 2.7$ & 0.01 \\
\hline & & Right & $15.1 \pm 2.7$ & $17.4 \pm 3.2$ & 0.02 \\
\hline & \multirow[t]{4}{*}{ Location } & \multirow[t]{2}{*}{ Left } & CPVC: $(11 / 17)$ & CPVC: (9/17) & NS \\
\hline & & & RBCPVC:(6/17) & RBCPVC:(7/17) & NS \\
\hline & & \multirow[t]{2}{*}{ Right } & CPVC: $(11 / 17)$ & CPVC: (10/17) & NS \\
\hline & & & RBcPVC: $(3 / 17)$ & RBCPVC: $(5 / 17)$ & NS \\
\hline
\end{tabular}

Abbreviations: $C P V C$ Contralateral primary visual cortex, RBCPVC Regions beyond contralateral primary visual cortex, NS Not significant

frequency range. Our results in the visual neuromagnetic field showed a comparatively normal low-frequency activation and, to a certain degree, aberrant high-frequency oscillation (HFO) in interictal female migraineurs.

The results of MEG waveforms revealed an obvious prolongation in the latency of VII in female migraine patients when compared to HC. Although the latency of VEP has been extensively studied, the results of these studies were contradictory across most VEP/VEFs observations [23]. The causes of the discrepancies were probably multifaceted, which may be largely accounted for by methodological differences (space frequency, reversal rate), patient's state (time interval to nearest attack), and nosological diversity [36]. Since the prolongation of VEP/ VEF latency was identifiable in other diseases, such as ophthalmopathy, stroke, Parkinson's disease [37, 38], this phenomenon seemed not to be migraine-specific, and therefore may not be an ideal diagnostic tool for migraine. Notably, a diffidence of VIIs latency and spectrum power between the left and the right hemisphere was observed in migraine subjects when compared to HC. Early VEPs studies also reported the asymmetrical responses in migraine patients $[23,39]$. It has been proposed that the susceptibility in different hemispheres of the migraine brain is heterogeneous. Unfortunately, the correlation between this phenomenon with the lateralization of pain was not analyzed in the current study because of the limited number of migraine subjects.

Our results from time-frequency analysis demonstrated that the activation pattern was similar between interictal migraine and $\mathrm{HC}$ in both low and high-frequency ranges.
When compared to our previous studies using motor and auditory modality [29, 31], the VEFs showed a normal presence at low frequency but reduced presence at high-frequency range either on the sensor level or source level. It is known that high-frequency oscillations (HFOs) can be detected from event-evoked potentials using bandpass filters as evidenced by a considerable sum of electrophysiology studies [40-42]. In the present study, we used a wide frequency range because high-frequency oscillation has been proposed to be associated with abnormal thalamocortical activities [40, 41], which is a pivot mechanism in the inhibitory pathway in migraine. In concord with these results, the present study also showed a diminution of spectral power in the high-frequency range in migraineurs when compared to HC. As mentioned above, spectral power in this study involves the sum of all cortical activation power detected on the sensor level, thereby representing the entire cortical activation. Interestingly, the low source strength was low in the occipital lobe from the low-frequency range, which seems to be paradoxical with a significantly high RMS value in migraineurs. The contradiction may possibly be due to the difference between the two methodologies, that is, the spectral power may contain noise from other regions of the brain while the noise was filtered on the source level. In the high-frequency range, however, spectral power was consistent with source strength. This phenomenon supports the notion that low-frequency signals might be produced by a large brain area whereas high-frequency brain signals are more likely produced by 
specific regions of the brain, thus are highly localized and may provide precise spatial information regarding cortical dysfunction [33, 43].

Our data is in favor of an interictal hypo-activation condition of the visual cortex in interictal female migraineurs in both the low and high-frequency range. These findings are in line with many VEP/VEF studies, thereby indicating a low initial amplitude of VEP/VEF in interictal migraineurs $[44,45]$. According to numerous electrophysiology studies, the excitability in the interictal migraine brain is better referred to as hyper-responsivity rather than hyperexcitability because the abnormality of the migraine brain involves primarily characteristics due to lack of habitation for a sensor stimulus $[12,36,44,46]$. In this study, we alternatively focused on the spatial-frequency trait, and source strength of the visual-evolved neuromagnetic responses especially the major component (VIIs) instead of the habituation process. Our data on source strength showed that the initial activation of VEF was low compared to that of $\mathrm{HC}$, which was in line with a low first VEP/VEF amplitude in other habitation studies. Low initial amplitude and a lack of habitation of event-related response (auditory, visual, somatosensory etc.) in migraineurs have been extensively reported in previous studies [13, 47, 48]. These reproducible abnormalities indicated a decreased pre-activation level of the cortical circle and increased neuronal hyperresponsivity in female migraineurs between attacks. High-frequency oscillations studies have shown that the hyperresponsivity and low preactivation in interictal migraine are associated with a dysfunctional thalamocortical afferent pathway may potentially contributed by aminergic neurotransmitter disposition [38-40].

Regarding source location, the data shown there was not diffident from the odds of activation in other brain areas other than the primary visual cortex in both the low and high-frequency range between the two groups. Migraine may be associated with visual-related network dysfunction [49], therefore, our initial hypothesis of this dysfunction of visual cortex in the migraine brain will evoke more extensive visual to participant in the response of visual stimulus. However, taking together the low source power results observed in migraine subjects, the phenomenon could be partly understood. The relation between visual cortex and pain perception is intriguing although the visual cortex is conventionally considered to be outside of pain matrix. On the one hand, tonic pain can change the excitability of the visual cortex in HC [50]. In their study, healthy subjects showed normal VEP patterns during baseline and no-pain conditions, while during pain and after-effects of tonic pain condition they showed identical VEP patterns as interictal migraineurs (low initial VEP amplitude and lack of habituation). On the other hand, the visual cortex may exert influence on the perception of pain, a potential top-down inhibitory pathway from the visual areas to trigeminal-cervical nociceptors may participant in the modulation of pain [51]. Especially, intra-individual correlations between dysfunction of visual and pain perception regions were found in migraineurs but not in $\mathrm{HC}$ [52]. Given that migraine is associated with the abnormal condition of subcortical/ intercortical inhibitory pathway $[46,53]$, the dual-directional regulation between visual cortex and pain will open a new window to the culprit of migraine.

In this study, a correlation between MEG results (latency, spectral power) and clinical parameters (i.e. intensity of headache, headache-history duration, frequency of headache attacks per month) was not observed, which is in line with data presented in many VEP/VEF studies [54]. Taken together, our finding supports the notion that subcortical/intercortical instead of cortical pathways were more likely to be the underlying mechanism for altered excitability in the visual cortex, which has been proposed by a large number of studies $[1,6]$. In a recent study using high frequency domains, it was suggested that thalamocortical dysrhythmia was also attributed to functional changes in the migraine brain [41], however, the exact mechanism of subcortical dysfunction still needs to be determined.

\section{Limitation}

Our study has some limitations. First, the sample size of $\mathrm{HC}$ is small, and patients with other subtypes of migraine (i.e. migraine with aura) were not enrolled in the study. Secondly, all participants are female, therefore the study needs to be extended to male migraineurs. Lastly, in this study, data from migraineurs during the ictal phase was not collected because participants may fail to cooperate during the scan with potential exacerbation by the visual stimulus. Therefore, a full picture of the periodic changes over the migraine cycle was not obtained in present study.

\section{Conclusions}

Neurological responses following a visual stimulus were abnormal in female migraine patients between attacks when compared to $\mathrm{HC}$. This difference was more significant in the high-frequency range.

\section{Abbreviations}

CPVC: Contralateral primary visual cortex; RBCPVC: Regions beyond contralateral primary visual cortex

Acknowledgements

Not applicable. 


\section{Funding}

This work was supported by the National Nature Science of China [grant numbers No.81271440]; "Key research And Development" project of Jiangsu province, China [grant numbers NO.BE2016615]; "High-level Talent" project of Jiangsu province, China [grant numbers NO. WSN-171]; "Six ones" elite project of Jiangsu province [grant numbers NO. LGY2017003]; Normal project of Nanjing [grant numbers NO. 201503040].

\section{Availability of data and materials}

The datasets used and during the current study are available from the corresponding author on reasonable request

\section{Authors' contributions}

Authorship are specified as following: XS contributed to the conception and design, QQ and T.W contributed to acquisition of data, D.W, YW and J.X contributed to analysis and interpretation of data; ZY. drafted the article and $\mathrm{HX}$ revised the article. All authors read and approved the final manuscript.

\section{Ethics approval and consent to participate}

This study was authorized by the Institutional Review Board of Nanjing brain hospital (NBH). All the participants endorsed written consent after being introduced to the whole procedure of the study.

\section{Consent for publication}

Not applicable.

\section{Competing interests}

The authors declare that they have no competing interests.

\section{Publisher's Note}

Springer Nature remains neutral with regard to jurisdictional claims in published maps and institutional affiliations.

\section{Author details}

${ }^{1}$ The Department of Neurology, Nanjing Brain Hospital, Nanjing Medical University, Guang Zhou Road 264, Nanjing, Jiangsu 210029, People's Republic of China. ${ }^{2}$ The MEG Center, Division of Neurology, Cincinnati Children's Hospital Medical Center, 3333 Burnet Avenue, Cincinnati, OH 45220, USA. ${ }^{3}$ The MEG Center, Nanjing Brain Hospital, Nanjing, Jiangsu, People's Republic of China.

\section{Received: 27 October 2018 Accepted: 27 December 2018}

Published online: 16 January 2019

\section{References}

1. Goadsby PJ, Lipton RB, Ferrari MD (2002) Migraine--current understanding and treatment. N Engl J Med 346(4):257-270

2. Leonardi M, Steiner TJ, Scher AT, Lipton RB (2005) The global burden of migraine: measuring disability in headache disorders with WHO's classification of functioning, disability and health (ICF). J Headache Pain 6(6):429-440

3. Lipton RB, Stewart WF, Diamond S, Diamond ML, Reed M (2010) Prevalence and burden of migraine in the United States: data from the American migraine study II. Headache 41(7):646-657

4. Stewart WF, Lipton RB, Celentano DD, Reed ML (1992) Prevalence of migraine headache in the United States. Relation to age, income, race, and other sociodemographic factors. JAMA 267(1):64-69

5. Steiner TJ, Scher Al, Stewart WF, Kolodner K, Liberman J, Lipton RB (2010) The prevalence and disability burden of adult migraine in England and their relationships to age, gender and ethnicity. Cephalalgia 23(7):519-527

6. Vecchia D, Pietrobon D (2012) Migraine: a disorder of brain excitatoryinhibitory balance? Trends Neurosci 35(8):507-520

7. May A (2009) New insights into headache: an update on functional and structural imaging findings. Nat Rev Neurol 5(4):199-209

8. Manzoni GC, Torelli P (2004) Headache classification: criticism and suggestions. Neurol Sci 25(3):s67-ss9

9. Wöber-Bingöl C, Wöber C, Karwautz A, Auterith A, Serim M, Zebenholzer K et al (2010) Clinical features of migraine: a cross-sectional study in patients aged three to sixty-nine. Cephalalgia 24(1):12-17

10. Shepherd AJ (2001) Increased visual after-effects following pattern adaptation in migraine: a lack of intracortical excitation? Brain 124(Pt 11):2310-2318
11. Chen WT (2011) Sustained visual cortex hyperexcitability in migraine with persistent visual aura. Brain A J Neurol 134(8):2387-95

12. Sand T, Zhitniy N, Stovner $L J$ (2008) Visual evoked potential latency, amplitude and habituation in migraine: a longitudinal study. Clin Neurophysiol 119(5):1020-1027

13. Schoenen J, Wang W, Albert A, Delwaide PJ (1995) Potentiation instead of habituation characterizes visual evoked potentials in migraine patients between attacks. Eur J Neurol 2(2):115-122

14. Di CLG, Magis D, Fumal A, De PV, Schoenen J (2010) Nociceptive blink reflex and visual evoked potential habituations are correlated in migraine. Headache 45(10):1388-1393

15. Hämäläinen M, Hari R, Ilmoniemi RJ, Knuutila J, Lounasmaa OV (1993) Magnetoencephalography — theory, instrumentation, and applications to noninvasive studies of the working human brain. Rev Mod Phys 65(2):413

16. Mosher JC, Leahy RM, Lewis PS (1999) Eeg and meg: forward solutions for inverse methods. IEEE Trans Biomed Eng 46(3):245-59

17. Nakamura A, Kakigi R, Hoshiyama M, Koyama S, Kitamura Y, Shimojo M (1997) Visual evoked cortical magnetic fields to pattern reversal stimulation. Brain Res Cogn Brain Res 6(1):9

18. Pitt MC, Daldry SJ (1988) The use of weighted quadratic regression for the study of latencies of the P100 component of the visual evoked potential. Electroencephalogr Clin Neurophysiol 71(2):150-152

19. Kunita K, Fujiwara K (2004) Changes in the P100 latency of the visual evoked potential and the saccadic reaction time during isometric contraction of the shoulder girdle elevators. Eur J Appl Physiol 92(4-5):421-424

20. Di-Russo F, Martinez A, Sereno M, Pitzalis S, Hillyard S (2010) Cortical sources of the early components of the visual evoked potential. Hum Brain Mapp 15(2):95-111

21. Kelly SP, Vanegas MI, Schroeder CE, Lalor EC (2013) The cruciform model of striate generation of the early VEP, re-illustrated, not revoked: a reply to Ales et al. (2013) is. Neurolmage 82(2):154-159

22. Ruchi K, Pradeep B, Smita S, Ramji S (2016) A comprehensive review on methodologies employed for visual evoked potentials. Scientifica 2016(1):1-9

23. Ambrosini A, Schoenen J (2006) Electrophysiological response patterns ofprimary sensory cortices in migraine. J Headache Pain 7(6):377-388

24. Huo X, Xiang J (2010) A MEG study of gamma oscillations during unilateral movement. Int Conf Bioinformatics \& Biomed Eng. IEEE.

25. Slater J, Thomas M, Hoskison M, Li Z, Castillo E, Papanicolaou A, editors. Detection of high-frequency oscillations in human epileptic brain using magnetoencephalography - revisited. American Epilepsy Society Meeting; 2008

26. Lu M, Xiang J (2015) Brain high frequency signal detection and localization based on MEG. J Med Imaging 5(8):1588-1591

27. Tepper SJ (2013) Editorial: international classification of headache disorders, 3rd edition, beta version. Cephalalgia 53(8):1381-1382

28. Wang X, Xiang J, Wang Y, Pardos M, Meng L, Huo X, Hershey AD (2010) Identification of abnormal neuromagnetic signatures in the motor cortex of adolescent migraine. Headache J Head Face Pain 50(6):1005-16

29. Xiang J, Degrauw X, Korostenskaja M, Korman AM, O'Brien HL, Kabbouche MA et al (2013) Altered cortical activation in adolescents with acute migraine: a magnetoencephalography study. J Pain 14(12): 1553-1563

30. Xiang J, Degrauw X, Korman AM, Allen JR, O"Brien HL, Kabbouche MA, et al. (2013) Neuromagnetic abnormality of motor cortical activation and phases of headache attacks in childhood migraine. PLoS ONE 8(12):e83669

31. Ge HT, Liu HX, Xiang J, Miao AL, Tang L, Guan QS et al (2015) Abnormal cortical activation in females with acute migraine: a magnetoencephalography study. Clin Neurophysiol 126(1):170-179

32. Xiang J, Leiken K, Degrauw X, Kay B, Fujiwara H, Rose DF, Allen JR, Kacperski JE, O'Brien HL, Kabbouche MA, Powers SW, Hershey AD (2015) Spatial heterogeneity of cortical excitability in migraine revealed by multifrequency neuromagnetic signals

33. Xiang J, Liu Y, Wang Y, Kirtman EG, Kotecha R, Chen Y et al (2009) Frequency and spatial characteristics of high-frequency neuromagnetic signals in childhood epilepsy. Epileptic Disord 11(2):113-125

34. Wheless JW, Castillo E, Maggio V, Kim HL, Breier JI, Simos PG et al (2004) Magnetoencephalography (MEG) and magnetic source imaging (MSI). Neurologist 10(3):138-153

35. Shigeto H, Tobimatsu S, Yamamoto T, Kobayashi T, Kato M (1998) Visua evoked cortical magnetic responses to checkerboard pattern reversal stimulation: a study on the neural generators of N75, P100 and N145. J Neurol Sci 156(2):186-194 
36. Sand T, ., Vingen JV. Visual, long-latency auditory and brainstem auditory evoked potentials in migraine: relation to pattern size, stimulus intensity, sound and light discomfort thresholds and pre-attack state. Cephalalgia 2010;20(9):804-820

37. He SB, Liu CY, Chen LD, Ye ZN, Zhang YP, Tang WG et al (2018) Metaanalysis of visual evoked potential and Parkinson's disease. Park Dis 2018: 3201308

38. Chahin S, Balcer L, Zarif M, Bumstead B, Fafard L, Mebrahtu S et al (2012) Visual evoked potential latency prolongation in MS: correlation with cognitive performance on a computerized testing battery (P07.260). Neurology 78:P07.260

39. Connolly JF, Gawel M, Rose FC (2010) Migraine patients exhibit abnormalities in the visual evoked potential. Headache J Head Face Pain 23(2):49-52

40. Coppola G, Vandenheede M, Clemente LD, Ambrosini A, Fumal A, De Pasqua V et al (2005) Somatosensory evoked high-frequency oscillations reflecting thalamo-cortical activity are decreased in migraine patients between attacks. Brain 128(Pt 1):98-103

41. Coppola G, Ambrosini A, Di Clemente L, Magis D, Fumal A, Gerard P et al (2007) Interictal abnormalities of gamma band activity in visual evoked responses in migraine: an indication of thalamocortical dysrhythmia? Cephalalgia 27(12):1360-1367

42. Adjamian P, Holliday IE, Barnes GR, Hillebrand A, Hadjipapas A, Singh KD (2004) Induced visual illusions and gamma oscillations in human primary visual cortex. Eur J Neurosci 20(2):587

43. Xiang J, Wang Y, Chen Y, Liu Y, Kotecha R, Huo X et al (2010) Noninvasive localization of epileptogenic zones with ictal high-frequency neuromagnetic signals. J Neurosurg Pediatr 5(1):113-122

44. Chen WT, Wang SJ, Fuh JL, Lin CP, Ko YC, Lin YY (2010) Peri-ictal normalization of visual cortex excitability in migraine: an MEG study. Cephalalgia 29(11):1202-1211

45. Boylu E, Domaç FM, Koçer A, Unal Z, Tanridağ T, Us O (2010) Visual evoked potential abnormalities in migraine patients. Electromyogr Clin Neurophysiol 50(6):303-308

46. Coppola G, Pierelli F, Schoenen J (2007) Is the cerebral cortex hyperexcitable or hyperresponsive in migraine? Cephalalgia 27(12):1427-1439

47. Ozkul Y, Uckardes A (2002) Median nerve somatosensory evoked potentials in migraine. Eur J Neurol 9(3):227

48. Wang W, Timsitberthier M, Schoenen J (1996) Intensity dependence of auditory evoked potentials is pronounced in migraine an indication of cortical potentiation and low serotonergic neurotransmission? Neurology 46(5):1404-1409

49. Granziera C, DaSilva AF, Snyder J, Tuch DS, Hadjikhani N (2006) Anatomical alterations of the visual motion processing network in migraine with and without aura. PLoS Med 3(10):e402

50. Coppola G, Serrao M, Currà A, Di LC, Vatrika M, Parisi V et al (2010) Tonic pain abolishes cortical habituation of visual evoked potentials in healthy subjects. J Pain 11(3):291-296

51. Sava SL, de Pasqua V, Magis D, Schoenen J (2014) Effects of visual cortex activation on the nociceptive blink reflex in healthy subjects. PLoS One 9(6): e100198

52. Clemente LD, Gianluca Coppola MD, Delphine Magis MD, Arnaud Fumal MD, Phy VDP, PhD JSM (2010) Nociceptive blink reflex and visual evoked potential habituations are correlated in migraine. Headache J Head Face Pain 45(10):1388-1393

53. Aurora SK, Barrodale P, Chronicle EP, Mulleners WM (2005) Cortical inhibition is reduced in chronic and episodic migraine and demonstrates a spectrum of illness. Headache 45(5):546-552

54. van Dijk JG, Dorresteijn M, Haan J, Ferrari MD (2010) Visual evoked potentials and background EEG activity in migraine. Headache J Head Face Pain 31(6):392-395

\section{Ready to submit your research? Choose BMC and benefit from:}

- fast, convenient online submission

- thorough peer review by experienced researchers in your field

- rapid publication on acceptance

- support for research data, including large and complex data types

- gold Open Access which fosters wider collaboration and increased citations

- maximum visibility for your research: over $100 \mathrm{M}$ website views per year

At BMC, research is always in progress.

Learn more biomedcentral.com/submissions 